\title{
Organización de la evaluación formativa y sumativa: una experiencia negativa
}

\author{
Fidel Salas Vicente ${ }^{1}$ y Ángel Vicente Escuder ${ }^{1}$ \\ ${ }^{1}$ Departamento de Ingeniería Mecánica y de Materiales. Universitat Politècnica de València. \\ Camino de Vera, s/n. 46022. Valencia
}

\begin{abstract}
The design of the systems of the formative and summative evaluation is of paramount importance to guide the students towards the achievement of the learning objectives that were planned in the course' syllabus and avoid, along with the appropriate motivation, passing the course being the goal of the students. That is not always easy and it is needed to know the behavior, not of the most gifted students, but of those less motivated.

In this paper we describe the unexpected and undesirable effects that an on-demand formative evaluation and the design of a summative evaluation that left one of its parts, problems resolving, to the end of the course, when, as there was no minimum mark in each part of the evaluation, almost all the students had practically passed the course. These facts led the students to center their effort in assure passing the course and not in achieving a good formation level and, with it, a good mark. Furthermore, if the problems part were eliminated, the marks would have been very high although the actual formation level of the students was low.
\end{abstract}

Keywords: formative evaluation, summative evaluation, motivation

\section{Resumen}

El diseño de los sistemas de evaluación formativa y sumativa es esencial para guiar a los alumnos hacia la consecución de los objetivos de aprendizaje planteados en la guía docente y evitar, junto con la motivación adecuada, que la meta de los alumnos se quede en la mera superación de la asignatura. Esto no siempre es fácil y precisa conocer cual es el comportamiento, no de los mejores alumnos, sino de los menos motivados.

En este trabajo se presenta el efecto negativo que ha tenido dejar el uso de la evaluación formativa a la voluntad de los alumnos y elegir una planificación para la evaluación sumativa que dejaba una parte, la resolución de problemas, para el final del curso, cuando, al no ser necesario obtener un mínimo en cada parte de la evaluación, casi todos 
los alumnos tenían prácticamente el aprobado. Esto ha llevado a los alumnos a centrarse en asegurar el aprobado y no en conseguir una buena formación y, en consecuencia, una buena calificación. Además, esta experiencia muestra que si la evaluación sumativa mediante problemas no se hubiese hecho las notas finales habrían sido muy elevadas aunque la formación real de los alumnos era baja.

Keywords: evaluación formativa, evaluación sumativa, motivación

\section{Introducción}

Tanto la docencia como los actos de evaluación deben planificarse cuidadosamente con el fin de que converjan los objetivos de alumnos y profesores, que no suelen ser exactamente los mismos:

- Alumno: Aprobar la asignatura, lo que le exige aprender.

- Profesor: Que el alumno aprenda, lo que le llevará a aprobar la asignatura.

Puede parecer sencillo compatibilizar ambos objetivos y consecuencias. Por un lado los alumnos buscarán aprobar (Meece, Blumenfeld y Hoyle 1988; McCoach y Siegle 2003) optimizando el uso de los recursos de que disponen -el tiempo es el más importante de todos ellos- no solo para terminar con éxito el curso sino también para atender todas las actividades extraescolares asociadas con su edad. Evidentemente, eso lleva a que se busque superar las asignaturas con el menor uso de recursos posibles, lo que permitirá liberar más cantidad para el resto de actividades. La visión de los docentes es distinta y más orientada a que los alumnos dediquen, de forma ordenada y continuada, tantos recursos como sean necesarios a su formación, pues eso asegurará su éxito académico, dejando los restos para otras actividades. Evidentemente, esta no es la única diferencia que se da. La edad, la posición y las experiencias vividas hacen que el enfoque que cada uno tiene ante el mismo problema sea muy distinto.

Como se ha comentado, unir ambas visiones no es fácil (Bardach y col. 2019) y requiere tanto que el docente entienda al alumno como que el alumno sea consciente de su responsabilidad. Son muchas las acciones que se pueden tomar por parte del profesor, pero una de las más importantes es el diseño de los procesos evaluativos, tanto formativos como sumativos (Dick 1977; Migueláñez 2009; Martínez Rizo 2013). Aunque ambos procesos podrían realizarse de la misma forma, la evaluación formativa está pensada para ofrecer a los alumnos información válida sobre el nivel alcanzado en su proceso de aprendizaje de forma que pueda corregir sus deficiencias y aprovechar sus puntos fuertes, mientras que la sumativa es la que se usará para dar una calificación final al final del curso.

Ya que superar la evaluación sumativa es la meta final del alumnado, es de especial interés plantearla de forma que lleve a los alumnos a centrarse continuamente en su propio proceso formativo y a no dejar para el último momento lo que puede ir haciendo a lo largo del curso (Pychyl 2000; Eilam y Aharon 2003). Son muchas las formas de hacerlo, y más aún con la aparición de nuevas metodologías docentes aparecidas al albor de los avances informáticos, y todas pueden ser válidas, tanto las "modernas" como las clásicas, pero en ningún caso hay que dejar de pensar en los alumnos que no sientan una gran motivación por los estudios académicos ni en que tanto la evaluación formativa como la sumativa deben cumplir su función.

En este caso se presentan los resultados obtenidos de un sistema evaluativo que ha cumplido a medias su función. Las pruebas formativas, basadas en tests y problemas breves disponibles online, 
han servido a los alumnos para mejorar sus notas en una parte de los exámenes sumativos, pero por otro lado no han sido adecuadamente usadas y han sido despreciadas en otra parte. Por su parte, el diseño de los exámenes sumativos ha sido una de las causas del bajo aprovechamiento de las pruebas formativas y de que los alumnos hayan centrado su atención solo en una parte de la asignatura, pero también han sido la herramienta imprescindible que ha permitido detectar los fallos del sistema.

\section{Objetivos}

El objetivo del presente trabajo es descubrir los errores que se han cometido al diseñar los procesos de evaluación formativa y sumativa en una asignatura de ciencia de materiales con el fin de proponer las correcciones necesarias que lleven a los alumnos a alcanzar los objetivos de aprendizaje y competencias previstos.

\section{Desarrollo de la innovación}

La asignatura de Materiales del Grado en Ingeniería en Diseño Industrial y Desarrollo de Productos, impartida en la Escuela Técnica Superior de Ingeniería del Diseño de la Universitat Politècnica de València, se divide en 14 unidades temáticas. Durante el curso 2019/2020 se ha cambiado la evaluación, tanto formativa como sumativa de la asignatura. Los cambios se resumen a continuación.

Evaluación sumativa en años anteriores:

- $15 \%$ : Exámenes online con 10 preguntas de tipo test. Uno para cada tema (15 en total).

- 15\%: Exámenes online consistentes en un problema, cuya resolución se envía escaneada o fotografiada. Uno por cada tema (15 en total).

- 55\%: Exámenes presenciales con 10 problemas para resolver. Se hacen 2 parciales.

- $15 \%$ : Memorias de las prácticas de laboratorio (15 sesiones) y tres exámenes online de tipo test.

- No hay nota mínima en ninguna de las partes evaluables.

Evaluación formativa en años anteriores:

- Los propios exámenes online de tipo test usados para la evaluación sumativa sirven también para la evaluación formativa de los alumnos.

Evaluación sumativa en el curso 2019/2020:

- 35\%: Exámenes online con preguntas de tipo test. Se hacen 2 parciales.

- $40 \%$ : Examen presencial con 10 problemas para resolver. Se hace 1 único examen al final del curso, en un acto evaluativo separado y tras los tests.

- 25\%: Memorias de las prácticas de laboratorio. 14 sesiones. 
- No hay nota mínima en ninguna de las partes evaluables.

Evaluación formativa en el curso 2019/2020:

- Se pone a disposición de los alumnos una serie de exámenes online tipo test y de resolución de problemas que son corregidos de forma automática y dan de forma inmediata al alumno la evaluación formativa que precisan.

En este punto hay que destacar que el hecho de que no exista una nota mínima exigible en las distintas partes evaluadas para poder superar el curso y la cantidad de sistemas de evaluación responde a la normativa de la escuela

Los tests formativos online constan de 10 preguntas de respuesta múltiple, de completar, relacionar o similares en los que solamente una es respuesta es correcta y no hay límite de tiempo. Cada vez que se realiza un test las preguntas se escogen aleatoriamente de una base de datos preparada en años anteriores, de forma que algunas preguntas pueden repetirse en varios tests. La base de datos contiene entre 30 y algo más de 100 preguntas para cada uno de los temas de la asignatura. Estos tests están accesibles al alumno de forma continuada a lo largo del curso. Una vez enviado el alumno recibe inmediatamente la nota obtenida junto con la respuesta correcta a las preguntas erradas.

En los exámenes formativos online que consisten en la resolución de un problema el alumno escribe el resultado que obtiene, que es automáticamente comparado con el resultado correcto, incluyendo un margen de error. En este caso el alumno, aunque recibe el resultado correcto no recibe información sobre el proceso de resolución.

Con el fin de estudiar el efecto que han tenido los cambios realizados en la metodología se han analizado las notas obtenidas por los alumnos y los datos que recoge la plataforma educativa de la Politécnica de Valencia, PoliformaT, cada vez que se hace una prueba online. Esto ha permitido determinar cual ha sido el comportamiento de los alumnos en cuanto a sus hábitos de estudio y compararlos con las calificaciones obtenidas.

\section{Resultados}

\subsection{Evaluación formativa}

Los alumnos hicieron un total de 20358 test online a lo largo del curso, aunque concentrados en los días previos al examen, tal y como muestra la Figura 1. Esta circunstancia implica que los alumnos no han hecho uso de los tests como herramienta de autoevaluación formativa con el fin de corregir sus deficiencias y mejorar su proceso de aprendizaje, sino que los han empleado como mera herramienta de preparación para los exámenes sumativos de tipo test. La gran cantidad de tests realizados indica que el objetivo ha sido ver la mayor cantidad de preguntas posibles. Como el número y variedad de preguntas que pueden ponerse para cada tema es, al final, limitado si no se tienen en cuenta las variantes sobre una misma cuestión, esta no es una mala estrategia para mejorar la calificación obtenida. Un asunto distinto es si esto también les ayuda a entender la materia estudiada.

En cuanto a los problemas online (Figura 2), la cantidad que hicieron los alumnos fue muchísimo menor, 1205 en total, lo que supone menos de un $6 \%$ de los tests que se hicieron. En este caso los 


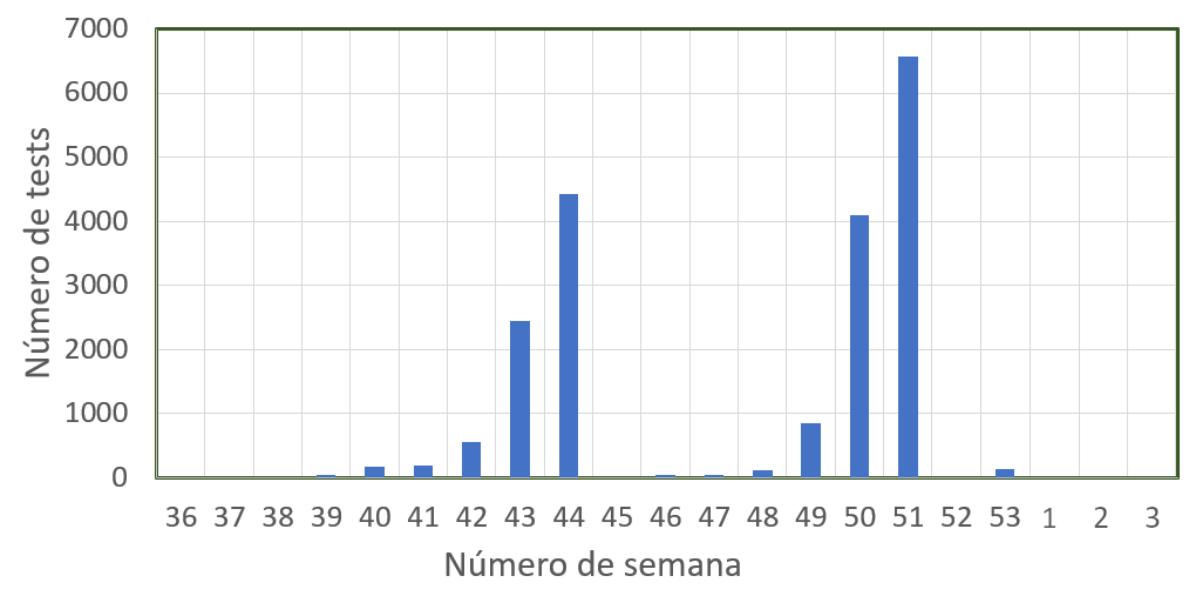

Fig. 1: Número de tests realizados por los alumnos en función del número de semana del año.

problemas hechos también se concentran mayoritariamente junto en las semanas antes del examen de problemas, que fue el último de todos y se realizó en Enero

Si se hace un cálculo en función del número de alumnos tenemos 155 tests resueltos por alumno frente a 9 problemas hechos por alumno, lo que indica que muchos ni siquiera llegaron a hacer un problema por cada uno de los temas.

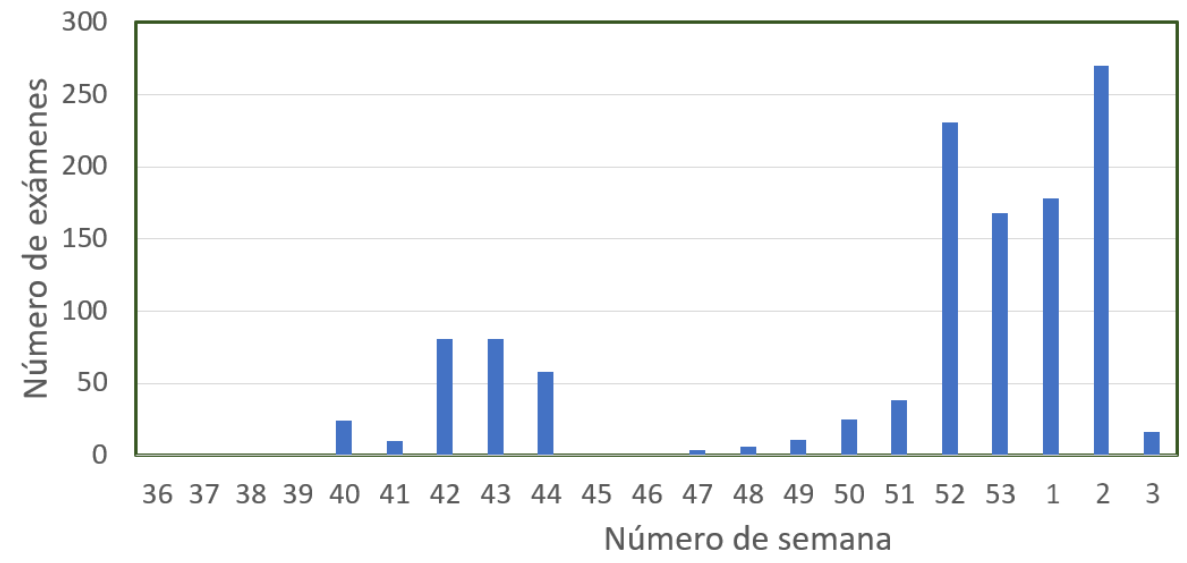

Fig. 2: Número de problemas online realizados por los alumnos en función del número de semana del año.

Si además se compara la nota media obtenida en los tests (varía entre 0 y 10) con la nota media obtenida en los problemas (como solo hay 1 problema por prueba, la nota es 0 o 10), la diferencia vuelve a ser enorme: 8,42 en los tests frente a 2,57 sobre 10 en los problemas, o dicho de otra forma, solo un $25,7 \%$ de los problemas que se intentaron resolver fueron resueltos correctamente. Este es un dato de suma importancia que requiere un análisis más profundo, sobre todo debido a que la resolución de un problema requiere poner en acción una serie de procesos cognitivos más amplios y complejos que contestar a una pregunta de tipo test o similar. 
En la asignatura se dedicó parte del tiempo presencial a la resolución de problemas, e incluso se había editado recientemente un libro de problemas adaptado a este curso, por lo que no es aceptable que se piense que no se haya prestado atención a la resolución de problemas por parte de los docentes y que el alumno no supiese que al final los problemas supondrían un $45 \%$ de la nota final. Otra cosa es si los alumnos han entendido que la resolución de problemas es una parte más importante incluso que los tests de cara a comprender la materia objeto de estudio.

\subsection{Evaluación sumativa}

La Figura 3 muestra las notas obtenidas por los alumnos en los 2 exámenes parciales de tipo test y en el examen de problemas.

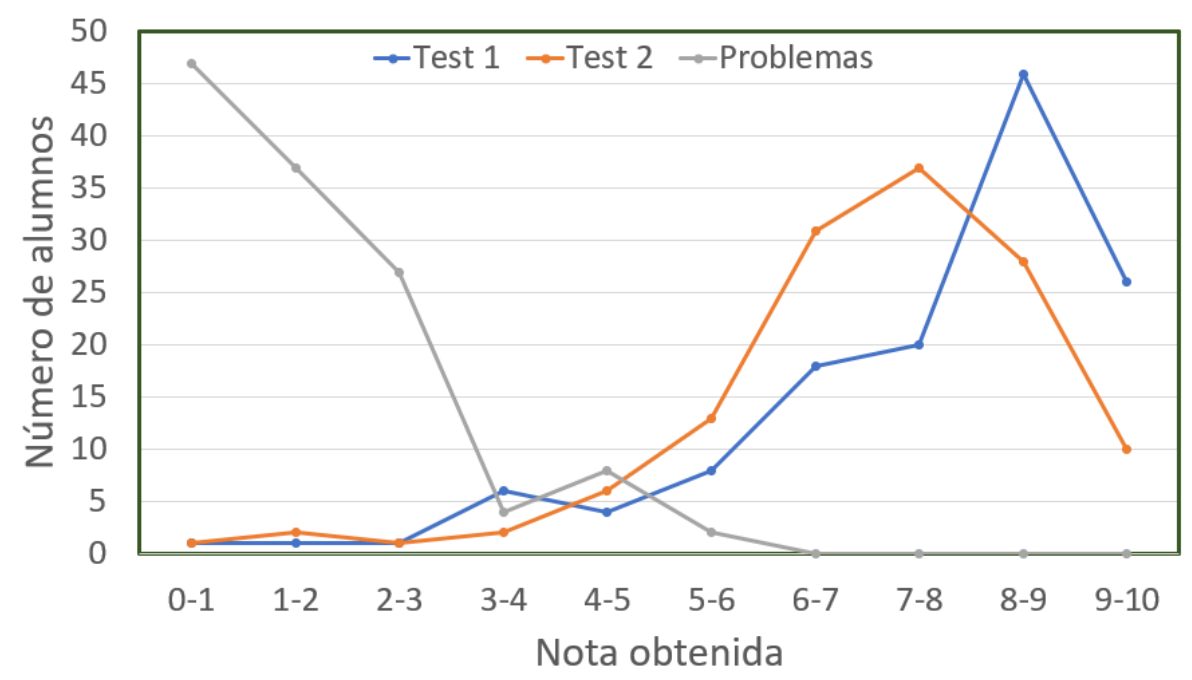

Fig. 3: Cantidad de alumnos que han obtenido una determinada nota en los distintos exámenes sumativos.

Como puede verse, la mayor parte de alumnos han obtenido una nota en los exámenes tipo test bastante buena, sobre todo en el primer parcial. En comparación, la nota media obtenida en el examen de problemas es extremadamente baja, sin que los docentes consideren que ese examen fuese particularmente complejo ni diferente a los que se ha hecho otros años (el año anterior la nota media fue cercana a 8). Esto sigue la tendencia vista en los exámenes formativos, con una clara preferencia por parte de los alumnos por prepararse para los exámenes tipo test.

En cuanto a la nota media final, ésta ha sido de 5,55 cuando en años anteriores se ha situado entre 6,5 y 7 . Esta media indica que otros años, los alumnos obtuvieron una nota media inferior en los tests (no disponían de los test online para practicar) y una nota notablemente superior en la parte de problemas, ante la cual este curso no se han tenido que enfrentar hasta el final.

Son varias las circunstancias que se juntan para dar lugar a este resultado:

- Los test son más rápidos de hacer y requieren menos esfuerzo mental en el sentido de que las preguntas se toman de una base de datos preparada para cada tema. Al hacer varios tests las preguntas acaban repitiéndose y, como el alumno conoce la respuesta, obtiene una falsa sensación de seguridad que no obtiene con los problemas. Además, en los problemas 
el alumno no obtiene al enviar un problema mal resuelto una información que le ayude a resolverlo correctamente a menos que contacte con el profesor, cosa que no suelen hacer.

- La inmensa mayoría de las pruebas formativas realizadas por los alumnos se concentran en los días anteriores a un examen, lo que no ayuda a llevar un seguimiento adecuado de la asignatura a lo largo del curso.

- El examen de problemas solo se hizo en el segundo parcial y fue el último de todos. Este es quizá uno de los puntos más importantes de todos pues los alumnos ya conocían la nota obtenida en el resto de procesos evaluativos, por lo que sabían si necesitaban obtener una buena nota o no en el examen de problemas para llegar al aprobado. En la mayor parte de los casos, debido a las notas más altas obtenidas en prácticas y en los exámenes de tipo test, tenían el aprobado prácticamente garantizado.

- No había nota mínima en ninguna de las partes evaluadas. Esto implica que si la suma de la nota de prácticas y tests ya llegaban al 5 no necesitaban ni presentarse al examen de problemas y mucho menos obtener una buena nota para aprobar. Más si cabe si durante el curso no han tenido la necesidad de hacer problemas, puesto que no se les ha evaluado esa parte hasta el final.

Todo esto es lo que ha llevado a que los alumnos se hayan centrado en los tests y no hayan prestado atención a los exámenes hasta el último momento. Como en ese momento ya no necesitaban esforzarse para aprobar la asignatura, puesto que la mayoría la tiene aprobada, tampoco se presta atención a los exámenes.

Las consecuencias de este comportamiento son extremadamente importantes si se atiende al proceso formativo de los alumnos. Por un lado evidencia la necesidad de guiarlos continuamente y, de alguna manera, obligarlos a asumir su propia responsabilidad en el proceso educativo a lo largo de todo el curso. Por otro lado muestra las consecuencias que puede tener un cambio en los métodos de evaluación si no se tiene cuidado. En este caso la mayor parte de los alumnos superaron el curso, aunque con una nota muy baja (vistos los resultados del examen de problemas, los docentes no consideran que hayan demostrado haber adquirido los conocimientos mínimos requeridos), pero si no se hubiese hecho el examen de problemas, el resultado habría sido muy distinto, con notas mucho más elevadas para todos ellos y este trabajo podría haberse presentado con unas conclusiones completamente distintas, aunque se habría pervertido la función de la evaluación sumativa.

De cara a próximos cursos es preciso conseguir que se haga un uso correcto de la evaluación formativa, quizá dejando disponibles los tests solamente durante un tiempo para evitar que los dejen para el final. Además, debe evitarse que los alumnos pierdan el interés por la asigantura al saber que ya están aprobados antes de finalizar las pruebas evaluativas, posiblemente no entregando notas parciales de los exámenes antes de que se hayan completado los actos evaluativos. Evidentemente, la exigencia de una nota mínima en cada una de las partes resolvería muchos de estos problemas. 


\section{Conclusiones}

Los procesos evaluativos, tanto formativos como sumativos se diseñan con un objetivo, que es facilitar la formación de alumno en un caso y comprobar el nivel de formación alcanzado en el otro. Si no se tiene el cuidado necesario es posible que dejen de cumplir su función y se pervierta el sistema educativo, dando lugar a muchos alumnos aprobados, incluso con notas altas, pero con escasa preparación.

En este caso se han identificado varios errores que han llevado a una falta de motivación en los alumnos para estudiar de forma correcta la asignatura. Por una parte se ha dejado a la elección de los alumnos el uso de las pruebas formativas, con la consecuencia de que no han usado de ellas hasta unos días antes de los exámenes y no a lo largo del curso, además de que se han centrado en la parte que más eficientemente podía facilitarles el aprobado. Por otra parte, quizá el error más importante de todos, se ha optado por la evaluación sumativa mediante tests en los dos parciales y no se usado la evaluación sumativa mediante la resolución de problemas hasta el final del curso, cuando la mayoría de ellos ya sabía que lo había superado. Estas deficiencias deberán corregirse en cursos posteriores consiguiendo que los alumnos usen la evaluación formativa a lo largo del curso y evitando que dispongan de un aprobado antes de terminar los actos evaluativos.

\section{Referencias bibliográficas}

Bardach, Lisa y col. (2019). "Students' and teachers' perceptions of goal structures - Will they ever converge? Exploring changes in student-teacher agreement and reciprocal relations to self-concept and achievement". En: Contemporary Educational Psychology 59, pág. 101799. DOI: 10.1016/j . cedpsych.2019.101799.

Dick, Walter (1977). "Summative evaluation". En: Instructional design: Principles and applications, págs. 337-348.

Eilam, Billie e Irit Aharon (2003). "Students' planning in the process of self-regulated learning". En: Contemporary Educational Psychology 28.3, págs. 304-334. DOI: 10.1016/s0361-476x (02) 000425 .

Martínez Rizo, Felipe (2013). "Dificultades para implementar la evaluación formativa: Revisión de literatura". En: Perfiles educativos 35.139, págs. 128-150.

McCoach, D. Betsy y Del Siegle (2003). "Factors That Differentiate Underachieving Gifted Students From High-Achieving Gifted Students". En: Gifted Child Quarterly 47.2, págs. 144-154. DOI: 10.1177/001698620304700205.

Meece, Judith L, Phyllis C Blumenfeld y Rick H Hoyle (1988). "Students' goal orientations and cognitive engagement in classroom activities." En: Journal of educational psychology 80.4, pág. 514.

Migueláñez, Susana Olmos (2009). "Evaluación formativa y sumativa de estudiantes universitarios: aplicación de las tecnologías a la evaluación educativa". En: Teoría de la Educación. Educación y Cultura en la Sociedad de la Información 10.1, págs. 305-307. 
Pychyl T A;Morin, R W;Salmon B R (2000). "Procrastination and the Planning Fallacy: An Examination of the Study Habits of University Students". En: Journal of social behavior and personality. 15.5, pág. 135. ISSN: 0886-1641. 